\title{
MULTIWAVELENGTH OBSERVATIONS OF PKS 0528+134 IN QUIESCENCE
}

\author{
MARKUS BÖTTCHER \\ Astrophysical Institute, Department of Physics and Astronomy \\ Ohio University, Athens, $\mathrm{OH}$ 45701, USA \\ boettchm@ohio.edu \\ NORMAN PALMA* \\ Astrophysical Institute, Department of Physics and Astronomy \\ Ohio University, Athens, OH 45701, USA \\ norman_palma@yahoo.com
}

\begin{abstract}
We present results of multiwavelength observations of the ultraluminous radio-loud quasar PKS 0528+134 in quiescence in the Fall of 2009. Significant flux variability on a time-scale of several hours was found in the optical regime, accompanied by a weak trend of spectral softening with increasing flux. The optical flux is weakly polarized with rapid variations of the degree and direction of polarization. Optical spectropolarimetry suggests a trend of increasing degree of polarization with increasing wavelength. Together with the spectral variability, this provides evidence for an unpolarized, slowly variable emission component, possibly thermal emission from the accretion disk, contributing towards the blue end of the optical spectrum.

We find that even in the quiescent state, the bolometric luminosity of PKS 0528+134 is dominated by its $\gamma$-ray emission. A leptonic single-zone jet model produced acceptable fits to the SEDs with contributions to the high-energy emission from both synchrotron self-Compton radiation and Comptonization of direct accretion disk emission. The moderate variability on long time scales, compared to the expected radiative cooling time scales, implies the existence of on-going particle acceleration, while the observed optical polarization variability seems to point towards a turbulent acceleration process.
\end{abstract}

Keywords: galaxies: active — galaxies: jets — gamma-rays: galaxies — radiation mechanisms: non-thermal — relativistic processes

\section{The Quasar PKS $0528+134$}

PKS $0528+134(z=2.07)$ is one of the most luminous and most distant $\gamma$-ray blazars known. In the $\gamma$-ray band, this source was detected by all three pointing telescopes onboard $C G R O^{1,2}$. During the EGRET era, PKS 0528+134 showed intense variability $^{3}$ exhibiting several strong flares. Its highest $\gamma$-ray flux was detected in 1993 March, when it reached $10^{14} \mathrm{JyHz}^{4}$, strongly dominating the bolometric

\footnotetext{
${ }^{*}$ Current address: Facultad de Ciencias Espaciales, Universidad Nacional Autonoma de Honduras, Tegucigalpa M.D.C., Honduras C.A.
} 
luminosity of the source. In the radio regime, PKS $0528+134$ shows pronounced variability on timescales of several months to a few years ${ }^{5,6,7}$. Increasing activity in the radio and $\gamma$-ray bands is associated with morphological changes in the radio structure of the jet, and superluminal motion with $\beta_{\perp, \text { app }} \lesssim 30$ is found in some of the jet components ${ }^{8}$.

Given the extreme properties of PKS 0528+134, this blazar has been the target of many observations at different wavelengths. However, as for almost all blazars, coordinated multiwavelength campaigns have targeted flaring states. The quiescent-state SEDs and spectral variability patterns of blazars in general, and of PKS $0528+134$ in particular, are poorly understood. Given its extended $\gamma$-ray quiescence throughout $2009^{\mathrm{a}}$, PKS $0528+134$ was therefore found to be an appealing target for our pre-approved XMM-Newton Cycle 8 ToO observations and multiwavelength campaign.

\section{Observations}

The core element of our campaign were four ToO pointings of $\sim 30 \mathrm{ksec}$ each, during the period 2009 September 8 - 14. These were supplemented by several ground-based radio and optical observations by members of the Whole Earth Blazar Telescope (WEBT) collaboration, publically available optical photometry from SMARTS $^{\mathrm{b}}$, as well as optical photometry, polarimetry, spectropolarimetry, and spectroscopy from the University of Arizona Fermi support program ${ }^{c}$. Long-term X-ray coverage was provided by $R X T E$, and additional Suzaku data from observations in SeptemberOctober 2008 were included in our analysis as PKS 0528+134 appeared to be in a similar quiescent state as during our fall 2009 campaign. Continuous $\gamma$-ray coverage was provided by Fermi LAT. For details of the observations and the data analysis, see ref. 9 .

\section{Variability}

Figure 1a shows the light curves of PKS 0528+134 in $\gamma$-rays (Fermi LAT), X-rays (RXTE), optical (R-band), and radio, for the period 2009 June - December. The most rapid flux variability was visible in the optical, with variations on time scales of a few hours. Moderate variability on weekly time scales was seen in the X-ray and radio bands, while no evidence for variability was found in the Fermi light curve.

Neither the X-ray nor $\gamma$-ray observations revealed evidence for any spectral variability, and even the Suzaku observations from one year before the XMM-Newton pointings showed a spectrum consistent with the $X M M$ one, though at a substantially higher flux level. However, the optical data indicate a significant redder-whenbrighter trend, which could indicate a steady emission component becoming dominant towards the blue end of the spectrum (see Figure 2). This correlation holds

\footnotetext{
${ }^{a}$ see http://fermi.gsfc.nasa.gov/ssc/data/access/lat/msl_lc/

${ }^{b}$ http://www.astro.yale.edu/smarts/

${ }^{\mathrm{c}}$ http://james.as.arizona.edu/psmith/Fermi/
} 

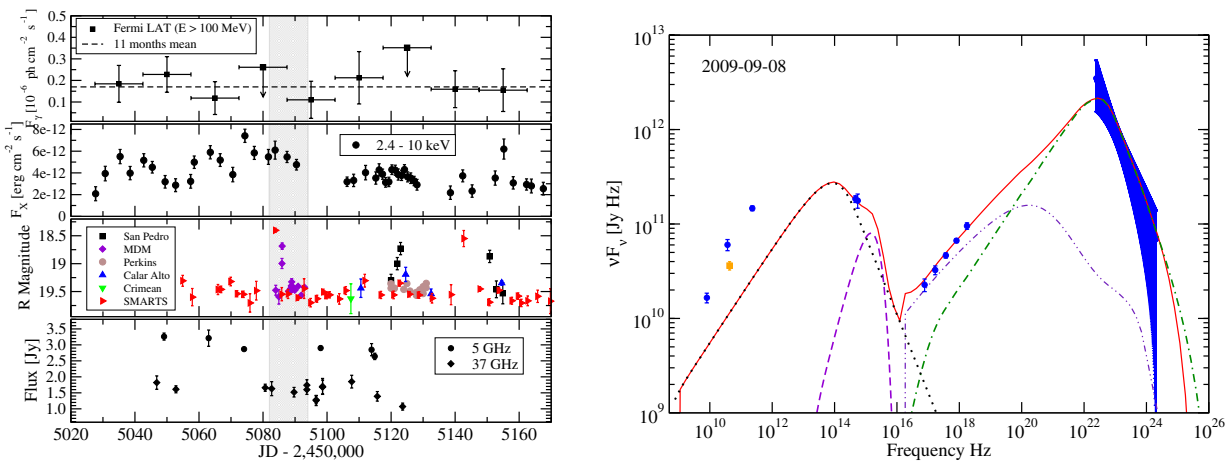

Fig. 1. Left panel (a): Multiwavelength light curves of PKS 0528+134 in the Fall of 2009. The gray shaded area indicates the core campaign period including the four XMM-Newton observations. Right panel (b): Spectral energy distribution of PKS 0528+134 of September12, 2009, including four spectral points from XMM-Newton. The SED can be fit with a leptonic-one-zone model with the $\gamma$-ray emission dominated by Comptonization of direct accretion disk emission.
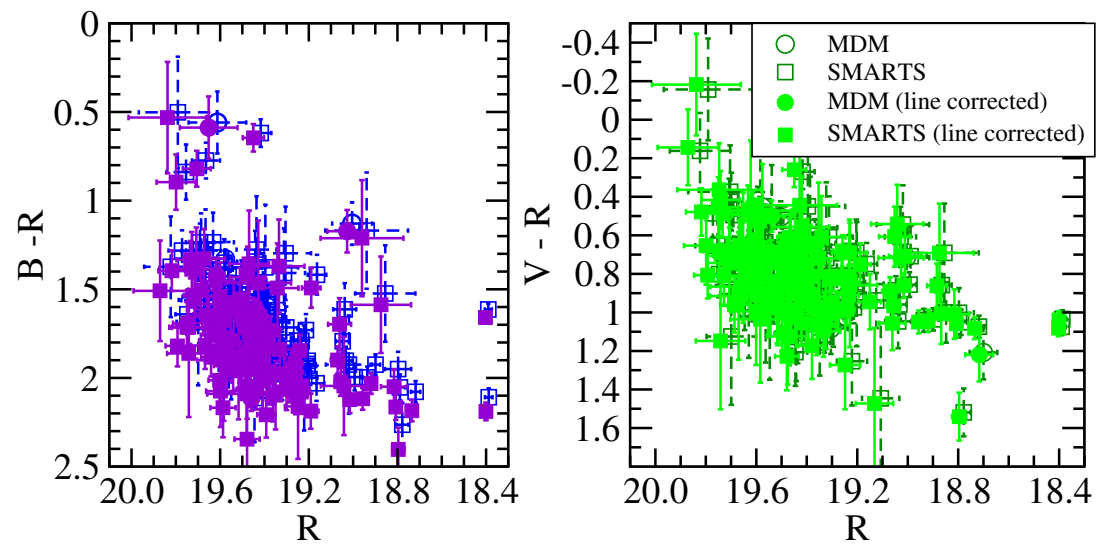

Fig. 2. Color-magnitude diagrams: B-R and V-R versus R-band magnitude. Open symbols show the correlations based on the raw (de-reddened) magnitudes, while filled symbols show the data corrected for the contributions from the CIV and CIII] emission lines.

even after correcting the B and V magnitudes for the (assumed steady) contribution from the red-shifted CIV and CIII] emission lines visible in the optical spectrum of PKS 0528+134 from the same epoch. Additional evidence for a steady, unpolarized emission component at the blue end of the optical spectrum comes from spectropolarimetry, which indicated a decrease of the degree of polarization towards higher frequencies. 
The optical emission of PKS $0528+134$ was polarized to $\lesssim 10 \%$, and the polarization angle varied substantially, with no apparent correlation with the optical flux. This might indicate that the optical emission originates in several turbulent cells with un-correlated magnetic-field orientations ${ }^{10}$.

\section{Spectral Energy Distribution}

The spectral energy distribution (SED) of PKS $0528+134$ during the first of our four XMM-Newton observations is shown in Figure 1b. It shows that even in its quiescent state, the SED of this quasar is strongly dominated by the $\gamma$-ray emission. The $\gamma$-ray spectrum measured by Fermi LAT (photon index $\Gamma_{\mathrm{ph}}=2.9 \pm 0.2$ ) during the quiescent state is substantially softer than the spectrum measured by EGRET in the 1990s $\left(\Gamma_{\mathrm{ph}} \sim 2.2-2.6^{11}\right)$. We modeled the SED using a single-zone leptonic jet model based on the code of Böttcher \& Chiang ${ }^{12}$. A good fit to the SED with parameters close to equipartition between the magnetic field and the relativistic electron population could be achieved with a scenario in which the X-ray emission is dominated by synchrotron-self-Compton emission, while the $\gamma$-rays are predominantly produced by Compton scattering of direct accretion disk emission. For more details of the analysis and interpretation, see ref. 9.

\section{Acknowledgments}

This work was supported by NASA through XMM-Newton Guest Investigator grant NNX09AV45G and Fermi Guest Investigator Grant NNX09AT82G.

\section{References}

1. J. R. Mattox et al., Astrophys. J. 481, 95 (1997).

2. K. McNaron-Brown et al., Astrophys. J. 451, 575 (1995).

3. B. L. Dingus et al., Astrophys. J. 467, 589 (1996).

4. R. Mukherjee et al., Astrophys. J. 470, 831 (1996).

5. H. D. Aller et al., Astrophys. J. Suppl. 59, 513 (1985).

6. W. Reich et al., Astron. Astrophys. 273, 65 (1993).

7. U. Bach et al., Astron. Astrophys. 464, 175 (2007).

8. S. Jorstad et al., Astron. J. 130, 1418 (2005).

9. N. Palma et al., Astrophys. J. 735, 60 (2011).

10. F. D. D'Arcangelo, Ph.D. Thesis, Boston University (2010).

11. R. Mukherjee et al., Astrophys. J. 527, 132 (1999).

12. M. Böttcher and J. Chiang, Astrophys. J. 281, 127 (2002). 